2000

\title{
Series Operation of Direct Current Xenon Chloride Excimer Sources
}

\author{
Ahmed El-Habachi \\ Old Dominion University \\ Wenhui Shi \\ Old Dominion University \\ Mohamed Moselhy \\ Old Dominion University \\ Robert H. Stark \\ Old Dominion University \\ Karl H. Schoenbach \\ Old Dominion University
}

Follow this and additional works at: https://digitalcommons.odu.edu/bioelectrics_pubs

Part of the Electrical and Electronics Commons, and the Physics Commons

\section{Repository Citation}

El-Habachi, Ahmed; Shi, Wenhui; Moselhy, Mohamed; Stark, Robert H.; and Schoenbach, Karl H., "Series Operation of Direct Current Xenon Chloride Excimer Sources" (2000). Bioelectrics Publications. 251.

https://digitalcommons.odu.edu/bioelectrics_pubs/251

\section{Original Publication Citation}

El-Habachi, A., Shi, W., Moselhy, M., Stark, R. H., \& Schoenbach, K. H. (2000). Series operation of direct current xenon chloride excimer sources. Journal of Applied Physics, 88(6), 3220-3224. doi:10.1063/1.1288699

This Article is brought to you for free and open access by the Frank Reidy Research Center for Bioelectrics at ODU Digital Commons. It has been accepted for inclusion in Bioelectrics Publications by an authorized administrator of ODU Digital Commons. For more information, please contact digitalcommons@odu.edu. 


\title{
Series operation of direct current xenon chloride excimer sources
}

\author{
Ahmed El-Habachi, Wenhui Shi, Mohamed Moselhy, Robert H. Stark, \\ and Karl H. Schoenbach ${ }^{\mathrm{a})}$ \\ Physical Electronics Research Institute, Department of Electrical and Computer Engineering, \\ Old Dominion University, Norfolk, Virginia 23529
}

(Received 20 March 2000; accepted for publication 16 June 2000)

\begin{abstract}
Stable, direct current microhollow cathode discharges in mixtures of hydrochloric acid, hydrogen, xenon, and neon have been generated in a pressure range of 200-1150 Torr. The cathode hole diameter was $250 \mu \mathrm{m}$. Sustaining voltages range from 180 to $250 \mathrm{~V}$ at current levels of up to $5 \mathrm{~mA}$. The discharges are strong sources of xenon chloride excimer emission at a wavelength of $308 \mathrm{~nm}$. Internal efficiencies of approximately 3\% have been reached at a pressure of 1050 Torr. The spectral radiant power at this pressure was measured as $5 \mathrm{~mW} / \mathrm{nm}$ at $308 \mathrm{~nm}$ for a $3 \mathrm{~mA}$ discharge. By using a sandwich electrode configuration, consisting of five perforated, alternate layers of metal and dielectric, a tandem discharge-two discharges in series-could be generated. For an anodecathode-anode configuration the excimer irradiance, recorded on the axis of the discharge, was twice as large as that of a single discharge. The extension of this basic tandem electrode structure to a multiple electrode configuration allows the generation of high irradiance excimer sources. Placing such a structure with a string of microhollow cathode discharge into an optical resonator promises to lead to a direct current microexcimer laser. (c) 2000 American Institute of Physics.
\end{abstract} [S0021-8979(00)07018-3]

\section{INTRODUCTION}

Microhollow cathode discharges (MHCDs) are highpressure gas discharges between a cathode, which contains a circular opening and an arbitrarily shaped anode. ${ }^{1}$ The diameter of the cathode hole as well as the electrode gap are in the $100 \mu \mathrm{m}$ range. Operation on such a small spatial scale allows us to sustain stable direct current glow discharge in rare gases even at high pressures. High-pressure operation favors excimer formation, a three-body process. The large concentration of high-energy electrons in MHCDs is essential for the generation of the precursors of the excimers, excited and ionized atoms. Experiments in xenon where emission of excimer radiation at $172 \mathrm{~nm}$, with an internal efficiency of approximately $8 \%$ has been recorded, ${ }^{2}$ have demonstrated the potential of these discharges as excimer light sources. Besides in xenon, excimer emission has been observed in argon at $130 \mathrm{~nm}$ (Refs. 1 and 3) and in neon at $84 \mathrm{~nm}^{4}$ MHCDs could even be operated dc in rare gas halide mixtures at up to atmospheric pressure. Such high-pressure MHCDs in $\operatorname{ArF}(1 \% \mathrm{~F}, 5 \% \mathrm{Ar}, 94 \% \mathrm{He})$ were found to be intense sources of excimer radiation at $193 \mathrm{~nm} .{ }^{5,6}$

The excimer radiant power of a discharge increases linearly with current. ${ }^{3}$ However, the excimer radiant emittance, defined as radiant power per emitting area, stays approximately constant due to the almost linear increase in the emitting area with current. ${ }^{6}$ One way to increase the radiant emittance is by increasing the pressure, as has been shown with discharges in xenon. ${ }^{7}$ However, this is a relatively weak effect: the radiant emittance increases only by a factor of 2 when the pressure is increased from 200 to 760 Torr. An-

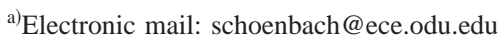

other method to increase the radiant emittance or the irradiance (defined as radiant power per target area), respectively, is based on the operation of an array of these discharges in series. Since the excimer gas does not reabsorb the excimer radiation, the excimer irradiance generated by $n$ discharge plasmas in series along the discharge axis is expected to be $n$ times that of a single discharge.

In order to study the physics of such strings of discharges, particularly coupling between discharges and discharge stability, we have used a three-electrode system, which allows us to generate two discharges in series, i.e., a "tandem" discharge. We have studied these discharges in $\mathrm{XeCl}$ mixtures, where the excimer wavelength is $308 \mathrm{~nm}$, rather than in xenon for a particular reason: the operation of MHCDs in series would open up the possibility of generating multiple excimer sources placed along a line between two mirrors, which could be used as medium for a dc excimer laser. Xenon chloride has the largest stimulated emission cross section and excimer lifetime of the rare gas halides and is therefore the best candidate for exploration of such an excimer laser concept.

\section{EXPERIMENTAL SETUP}

The electrode geometry consists of three molybdenum foils of 250,100, and $250 \mu \mathrm{m}$ thickness, respectively, separated by mica sheets of $200 \mu \mathrm{m}$ thickness. It is shown in the insets in all figures except Fig. 3. This electrode configuration allowed us to study both single discharges, with one electrode disconnected, and tandem discharges. A $250 \mu \mathrm{m}$ diam hole was drilled mechanically through the five layers. The electrodes could be biased in any way: it was possible to have the outer electrodes serving as cathodes with the inner electrode being the common anode or the other way around. 
The value of the dc discharge current was obtained by measuring the voltage across a current-viewing resistor of $1 \mathrm{k} \Omega$ in series with the discharge; the voltage was measured by means of a voltage probe attached to one of the electrodes.

Spectral measurements have been performed using a 0.5 $\mathrm{m}$ McPherson scanning monochromator, model 219, with a grating of $600 \mathrm{G} / \mathrm{mm}$ blazed at $150 \mathrm{~nm}$. The discharge chamber with a fused silica window was mounted directly to the inlet of the monochromator. Before each experiment, the chamber was evacuated to a pressure of $7 \times 10^{-4}$ Torr and then filled with a $\mathrm{XeCl}\left(1.5 \% \mathrm{Xe}, 0.03 \% \mathrm{HCl}, 0.06 \% \mathrm{H}_{2}\right.$, $98.41 \% \mathrm{Ne}$ ) mixture. The ultraviolet (UV) spectroscopic system is described in more detail in Ref. 2. In order to determine the absolute values of the excimer radiation we have compared the discharge emission with that of a deuterium lamp (Oriel BJ2775) with a factory calibrated spectrum from 200 to $400 \mathrm{~nm}$. The lamp was mounted directly to the inlet of the monochromator in the same way as the discharge chamber, and the same fused silica window as that in the discharge chamber was used. The efficiency was then calculated by dividing the measured UV optical power by the input electrical power, which is the product of the discharge current and voltage at a given pressure.

In addition to spectral measurements we have measured the spatial distribution of the excimer source by means of an UV imaging system. Excimer radiation was separated from all other radiation by using an UV filter with a peak transmittance at a wavelength of $307.3 \mathrm{~nm}$ and a full width at half maximum (FWHM) of $24.4 \mathrm{~nm}$. The UV image was recorded using an RS Princeton Instruments ICCD-MAX intensified charge coupled device (CCD) camera. The ICCD-MAX uses a proximity focused microchannel plate image intensifier fiber optically coupled to a CCD array.

\section{EXPERIMENTAL RESULTS}

By disconnecting one of the outer electrodes, the electrical and optical features of a single discharge in this tandem electrode configuration could be explored. Two modes of the microhollow cathode discharge were observed in this case depending on the polarities of the remaining outer electrode and the inner electrode. With the outer electrode serving as the cathode the $I-V$ characteristic of the discharge resembles that of a normal glow discharge (Fig. 1). This is in accordance with results which were previously obtained for xenon discharges. ${ }^{5,6}$ However, when the outer electrode is the anode, the $I-V$ characteristics have, except for low currents, a distinct resistive behavior (Fig. 2). The differential resistance of the discharge in this mode is on the order of $\mathrm{k} \Omega$. The lower current limit for the resistive mode depends on pressure: it is less for lower pressure. At high currents, currents exceeding 4-5 $\mathrm{mA}$ (depending on the pressure), the discharge tends to become unstable. Besides the $I-V$ characteristics, the excimer source has a quite different appearance for the two modes. In the case where the outer electrode is the anode, the excimer source is confined to the electrode opening (Fig. 3, left), whereas in the second case (the outer electrode cathode) it spreads over the outer electrode surface

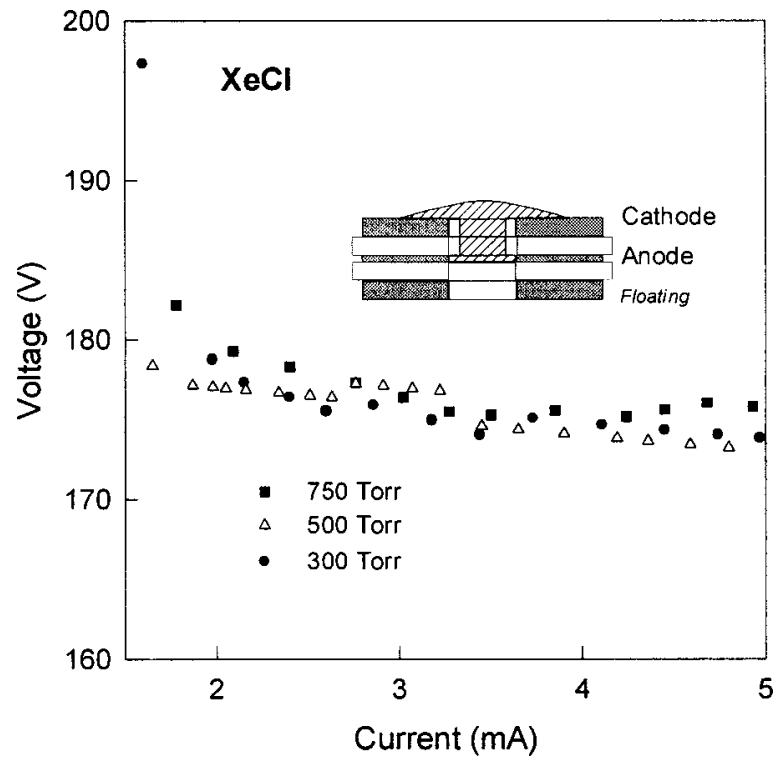

FIG. 1. Current-voltage characteristics of a single direct current discharge at various pressures in the electrode configuration shown in the inset. The center electrode is in this case the anode. The third electrode was disconnected. Also shown in the inset is the cross section of the discharge plasma, as estimated from UV end-on photographs (Fig. 3, right).

(Fig. 3, right). The excimer source in the latter case is generally not symmetric about the electrode opening and varies in shape and position.

The excimer spectrum of the $\mathrm{XeCl}$ radiation measured end on for the configuration where the inner electrode was the cathode is shown in Fig. 4 for various pressures. The main spectral feature is the $\mathrm{XeCl}$ excimer line at $308 \mathrm{~nm}$ corresponding to the $B-X$ band. The FWHM is $4 \mathrm{~nm}$. Measuring the emission end on from the opposite side gave the same value of radiant power. This indicates that the radiation

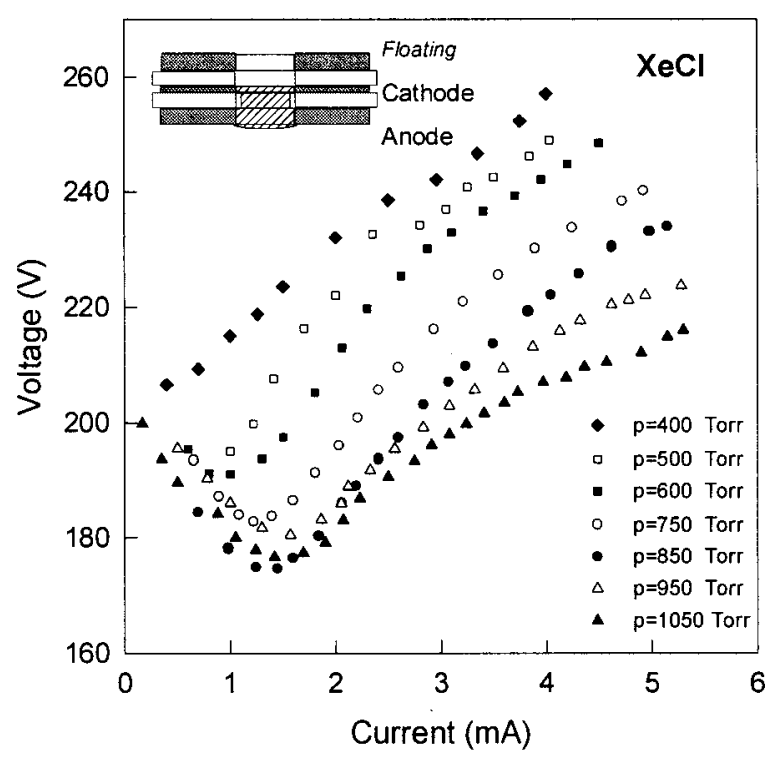

FIG. 2. Current-voltage characteristics of a single direct current discharge at various pressures in the electrode configuration shown in the inset. The center electrode is in this case the cathode. The third electrode was disconnected. Also shown in the inset is the cross section of the discharge plasma, as estimated from UV end-on photographs (Fig. 3, left). 

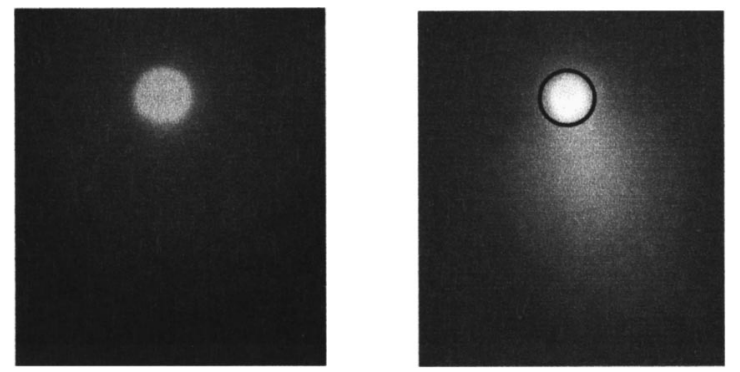

FIG. 3. End-on photographs of the discharges through a filter which only allowed xenon chloride excimer radiation to pass: at the left for the configuration with the outer electrode being the anode, and at the right for the configuration with the outer electrode being the cathode. The ring indicates the circumference of the electrode opening for the second case. In both cases the pressure was 1050 Torr, the current $4 \mathrm{~mA}$, and the sustaining voltage $200 \mathrm{~V}$. The direction of observation was from the top in the geometry shown in the insets in Figs. 1 and 2, respectively.

source is confined in the cathode hole. A similar pressure dependence of the excimer spectrum was obtained when the center electrode served as the anode. However, the values of the measured radiant power from the cathode side are higher than that recorded from the anode side. This is due to better optical access to the excimer light source, compared to the configuration where the inner electrode is the cathode. In the case where the outer electrode is the cathode the excimer emitting plasma expands over the cathode surface outside the hole (Fig. 3, right), whereas in the opposite case the excimer source is located inside the electrode hole, probably close to the center electrode. The efficiency of the excimer source, for the case where the center electrode is the anode, was calculated and is shown in Fig. 5 as a function of pressure. It increases with pressure and reaches a maximum of $3.2 \%$ at 1050 Torr. The spectral power at $308 \mathrm{~nm}$ for this pressure is $5 \mathrm{~mW} / \mathrm{nm}$ for a discharge current of $3 \mathrm{~mA}$.

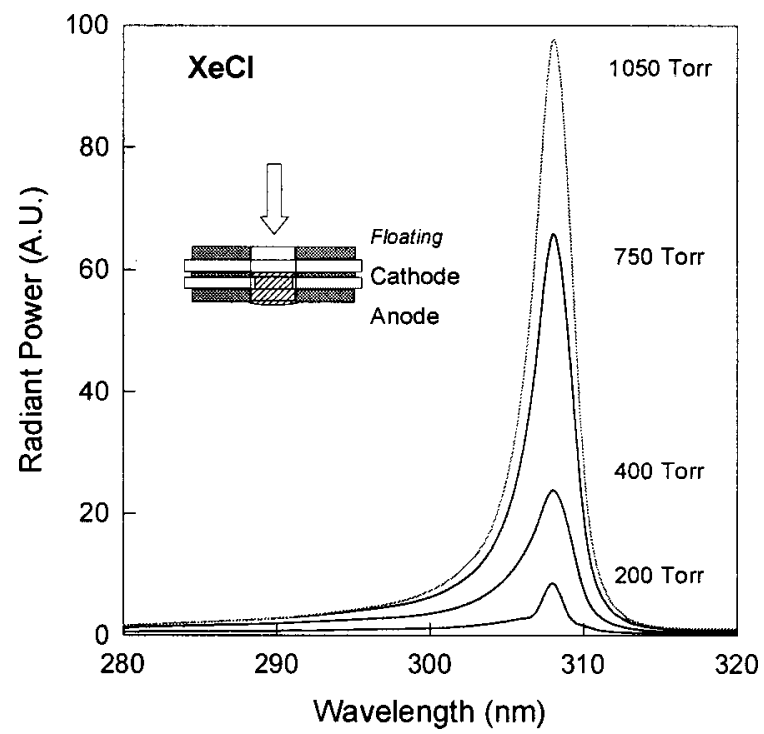

FIG. 4. Excimer spectra of a single discharge for various pressures. The discharge configuration (the center electrode is the cathode) is shown in the inset, along with a schematic sketch of the cross section of the discharge plasma. The arrow indicates the direction of observation.

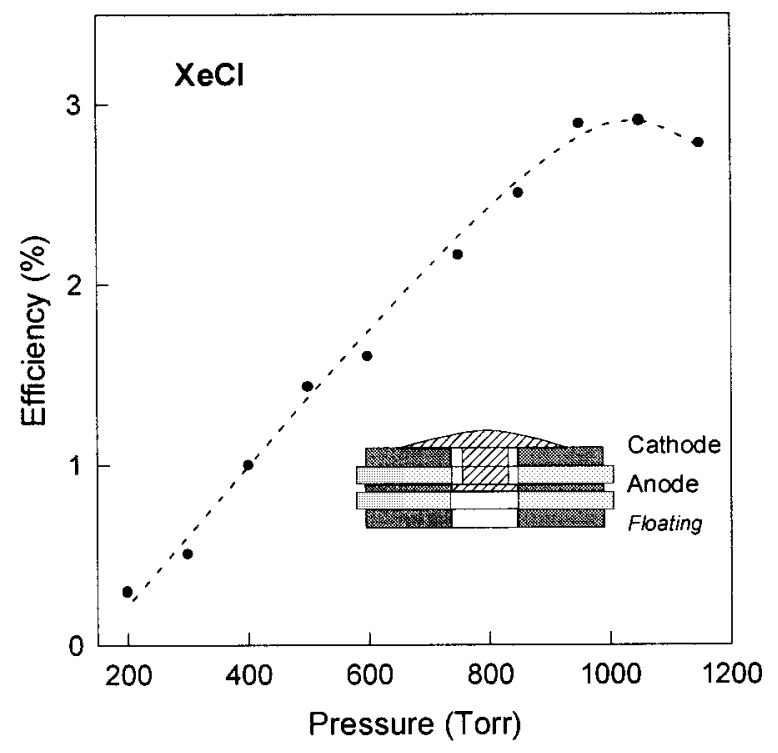

FIG. 5. Internal efficiency for xenon chloride excimer emission from a single discharge vs pressure. The discharge current was kept constant at 3 $\mathrm{mA}$. Also shown is the discharge configuration, with a schematic sketch of the discharge plasma.

For the case where the third electrode is also biased (rather than disconnected, as discussed so far) two basic configurations were studied: one where the outer electrodes were anodes (inset in Fig. 6), and the other with these electrodes being cathodes (inset in Fig. 7), respectively. In both cases we have recorded the spectrum of each individual discharge and the tandem discharge. The spectral distribution was

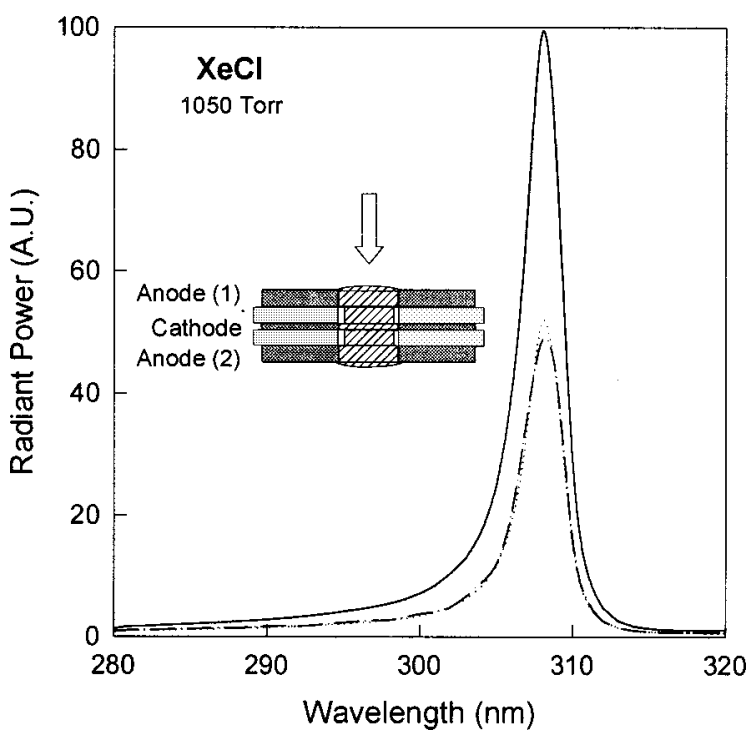

FIG. 6. Excimer spectra of single discharges between the center cathode and anode (1) (dotted line) and anode (2) (dashed line), and the combined (tandem) discharge (solid line). The electrode configuration with a schematic sketch of the discharge plasma and direction of observation (arrow) is shown in the inset. The current of the tandem discharge was $3.32 \mathrm{~mA}$ at a voltage of $190.7 \mathrm{~V}$, that of the single discharge (1) $1.7 \mathrm{~mA}$ at $170.5 \mathrm{~V}$, and that of the single discharge (2) $1.63 \mathrm{~mA}$ at $170.0 \mathrm{~V}$. The measurements were performed by subsequently disconnecting the outer electrodes (electrodes 1 and 2) to obtain the characteristics of the individual discharges. Then all three electrodes were biased to obtain the characteristics of the tandem discharge. 


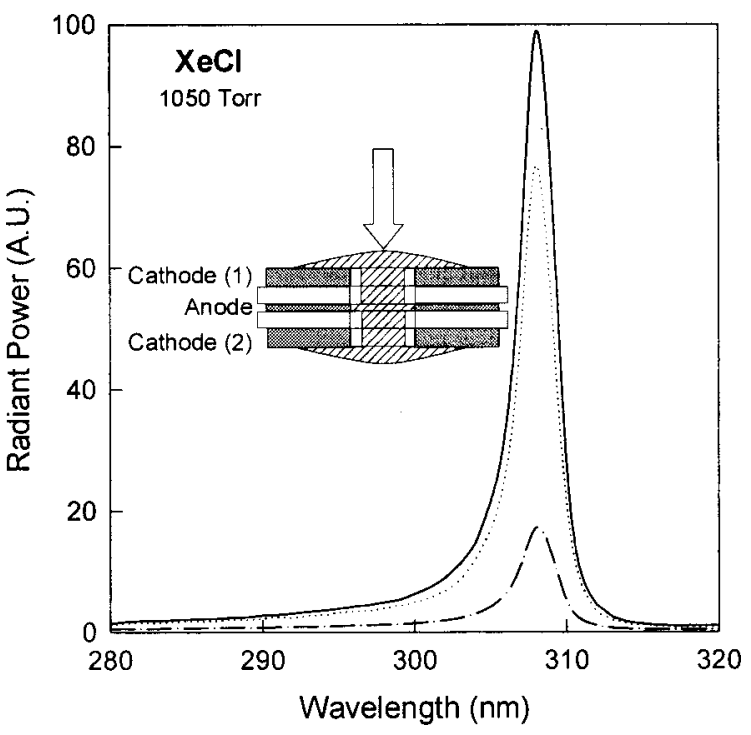

FIG. 7. Excimer spectra of single discharges between the center anode and cathode (1) (dotted line) and cathode (2) (dashed line), and the combined (tandem) discharge (solid line). The electrode configuration with a schematic sketch of the discharge plasma and direction of observation (arrow) is shown in the inset. The current of the tandem discharge was $6 \mathrm{~mA}$, equally distributed between the two discharges extending to the outer cathodes (by means of two external current sources connected to the cathodes). The sustaining voltages of the two discharges in the tandem configuration, however, differed: $219 \mathrm{~V}$ for discharge (1), $202 \mathrm{~V}$ for discharge (2). For the individual discharges the voltage was $200 \mathrm{~V}$, the current $3 \mathrm{~mA}$ [discharge (1)], and 197 $\mathrm{V}$ and $3 \mathrm{~mA}$ [discharge (2)], respectively. The measurements were performed in the same way as described in the caption of Fig. 6.

found to be identical to that of single discharges, however the amplitude was strongly dependent on the configuration.

Using the center electrode as the cathode, two stable discharges with almost identical current could be obtained between this electrode and the two outer anodes without ballasting each discharge individually. The spectral emission of the two discharges is of the same magnitude, as shown in Fig. 6. The sum of the radiant power of the two single discharges is, as expected (because of the negligible absorption of the excimer radiation), identical to that of the tandem discharge.

For the case where the center electrode is the anode, the current of the two discharges extending from this center electrode to the outer electrodes was not identical. In order to generate the same current in each of the two discharge branches the outer electrodes needed to be biased individually (by using two power supplies). The measured radiant power of the two individual discharges even for the same current differs in this configuration drastically. This is not surprising. The radiant power for the discharge between the cathode (1) and anode (inset in Fig. 7) is measured end on from the cathode side where the plasma expands over the cathode surface; consequently the total emission is recorded. The discharge between the cathode (2) and anode is recorded from the anode side where only $20 \%$ of the radiation is observable $(80 \%$ of the source extends over the outer cathode surface).

\section{DISCUSSION}

Stable dc operation of MHCDs in a gas mixture containing a strong attacher, $\mathrm{HCl}$, was achieved up to pressures of 1150 Torr. In this mixture which contained also xenon and hydrogen, with neon as a buffer gas, xenon chloride excimers were generated. The internal radiative efficiency reached a maximum value of $3.2 \%$ at a pressure of 1050 Torr. At this pressure, the radiant power is consequently 19 $\mathrm{mW}$ for a current of $3 \mathrm{~mA}$ and a sustaining voltage of $195 \mathrm{~V}$. The spectral peak power at $308 \mathrm{~nm}$ is $5 \mathrm{~mW} / \mathrm{nm}$. For the same input power this is higher by a factor of 2 compared to xenon excimer emitters, ${ }^{3}$ due to the small FWHM of the 308 xenon chloride line $(4 \mathrm{~nm})$ compared to that of the xenon line $(24 \mathrm{~nm})$.

The discharges were generated in a three-electrode sandwich arrangement, which allowed us to study both single discharges as well as tandem discharges, discharges arranged in series. The results of the measurements of the currentvoltage characteristics of the single discharges reflect the asymmetry in the electrode geometry. The center electrode is ring shaped with a limited surface defined by the hole radius and the foil thickness. It is therefore expected that the discharge for the configuration is, at high currents, an abnormal glow where the voltage increases with current. The outer electrode has a much larger surface, which includes the surface area of the outer electrode. In the configuration, where the outer electrode serves as the cathode, the cathode fall can expand over an almost unlimited area. The discharge in this case behaves as a normal glow discharge, with constant voltage at increasing current. The excimer source is always located at the cathode, as shown in the UV images of the plasma. This explains the differences in optical power observed from opposite end-on directions for different biasing.

For the tandem operation, with the center electrode being the cathode, the range of stable operation was limited to approximately $5 \mathrm{~mA}$. This is due to the onset of instabilities, possibly glow-to-arc transitions, which become more likely at higher cathode fall voltages. A way to increase the current range for stable operation is therefore to increase the cathode surface by using a thicker cathode foil. The anode area, as demonstrated by experiments in the configuration where the outer electrode serves as anode, does not limit the discharge stability, at least not in the current range, which was explored in our measurements.

The resistive current-voltage characteristics of the microdischarges in the configuration where the center electrode, with its limited surface, serves as the cathode indicate the possibility of forming longitudinal arrays consisting of a multielectrode sandwich structure with the metal foils (electrodes) biased alternately. The extension of the basic threeelectrode structure to a multiple sandwich structure provides an electrode configuration, which resembles a multitude of basic anode-cathode-anode structures, rather than cathodeanode-cathode structures, where control of the discharges would require current control at every electrode. In a multielectrode configuration all the electrodes, anodes and cathodes, would be limited in surface area, due to the dielectric spacers between them. The electrodes at both ends could be 
covered with a dielectric to prevent normal glow discharges even at the outer electrodes. Consequently any of the discharges in this multielectrode system would be abnormal, with resistive discharge characteristics (at higher currents), the condition for stable operation. The irradiance generated by such a discharge string on the axis of the hollow electrode configuration would for $n$ discharges be $n$ times that of a single discharge. This is due to the negligible reabsorption of excimer radiation in the excimer gas. Running these discharge strings in parallel will therefore allow us to generate more powerful flat panel excimer lamps compared to just using planar structures with single discharges arranged in parallel. ${ }^{8}$

A second application for a string of discharges would be as an excimer laser medium. The input electrical power for a single discharge is on the order of watts. The volume of the cathode fall and negative glow, where most of the electrical energy is dissipated, is assumed to be less than or at most equal to the volume of the cathode opening. For a $250 \mu \mathrm{m}$ thick, $100 \mu \mathrm{m}$ diam cathode hole the cathode hole volume is $1.9 \times 10^{-6} \mathrm{~cm}^{3}$. The power density in this plasma will therefore exceed $500 \mathrm{~kW} / \mathrm{cm}^{3}$. The typical fluorescence lifetime, $\tau$, of the $\mathrm{XeCl}$ excimer is $11 \mathrm{~ns}^{9}$, and the photon energy, $h \nu$, is $4 \mathrm{eV}$. The population density of the upper energy state $N_{2}$ is related to the spontaneous power density $P_{s}$ by

$$
N_{2}=\left(\frac{\tau}{e}\right)\left(\frac{e}{h \nu}\right) P_{s} .
$$

If we assume that it requires at least ten times the spontanous power density $\left(P_{s}=500 \mathrm{~kW} \mathrm{~cm}^{-3} / 10\right)$ to excite such a laser, $N_{2}$ would be $8.59 \times 10^{14} \mathrm{~cm}^{-2}$.

$$
k=\left(N_{2}-g_{2} N_{1} / g_{1}\right) \sigma_{\mathrm{se}} .
$$

Assuming that the lower state is empty $\left(N_{1}=0\right)$, and using the cross section for $\mathrm{XeCl}$ stimulated emission, $\sigma_{\text {se }}$, of $4.5 \times 10^{-16} \mathrm{~cm}^{2},{ }^{9}$ the small signal gain coefficient will then be $0.38 / \mathrm{cm}$.

With such gain conditions, lasing should be achievable if only sufficient discharges are placed along the line of sight. The advantages of such a laser, besides operating continuous wave (cw), would be its size which is expected to be in the $\mathrm{mm}$ range (depending on the number of discharges in series), the low sustaining voltage, which is comparable to that of a single discharge voltage, approximately $200 \mathrm{~V}$, and the possibility of creating an array of microlasers by placing them in parallel, similar to the lamp structure explored earlier. ${ }^{8}$

\section{ACKNOWLEDGMENT}

This work was supported by the National Science Foundation.

${ }^{1}$ K. H. Schoenbach, A. El-Habachi, W. Shi, and M. Ciocca, Plasma Sources Sci. Technol. 6, 468 (1997).

${ }^{2}$ A. El-Habachi and K. H. Schoenbach, Appl. Phys. Lett. 73, 885 (1998).

${ }^{3}$ A. El-Habachi and K. H. Schoenbach, Appl. Phys. Lett. 72, 22 (1998).

${ }^{4}$ P. Kurunczi, H. Shah, and K. Becker, J. Phys. B 32, L651 (1999).

${ }^{5}$ W. Shi, A. El-Habachi and K. H. Schoenbach, Bull. Am. Phys. Soc. 44, 25 (1999)

${ }^{6}$ K. H. Schoenbach, A. El-Habachi, M. Moselhy, W. Shi, and R. H. Stark, Phys. Plasmas 7, 2186 (2000).

${ }^{7}$ A. El-Habachi, M. Moselhy, and K. H. Schoenbach, Bull. Am. Phys. Soc. 44, 67 (1999).

${ }^{8}$ W. Shi, R. H. Stark, and K. H. Schoenbach, IEEE Trans. Plasma Sci. 27, 16 (1999).

${ }^{9}$ J. T. Verdeyen, Laser Electronics, 3rd ed. (Prentice-Hall, Englewood Cliffs, NJ, 1995). p. 414. 